\title{
Experimental Investigation of Vortex Structures in Wake of Hyperboloid-Shaped Model by Means of 2D Particle Image Velocimetry Measurement
}

\author{
V. Barraclough *, J. Novotný **, and P. Šafař́́k** \\ * Aerospace Research and Test Establishment, PLC. (ARTE), Prague, Czech Republic \\ ** Czech Technical University in Prague, Czech Republic
}

\begin{abstract}
This paper deals with flow around a bluff body of hyperboloid shape. It consists of results gathered in the course of research by means of Particle Image Velocimetry (PIV). The experiments were carried out by means of low-frequency 2D PIV in a range of Reynolds numbers from 40000 to 50000 . A hyperboloid-shaped model was measured in a wind tunnel with a modelled atmospheric boundary layer (and additionally, in a low-speed wind tunnel with low turbulence). The model was tested in a subcritical range of Reynolds numbers and various planes in a wake of the model were captured with the intention of getting an estimation of 3D flow structures. The tunnel with the modelled atmospheric boundary layer has a high rate of turbulence, so the influence of the turbulence of incoming flow on the wake could be outlined. The ratio of the height of the model to a thickness of the modelled boundary layer in the tunnel was $1 / 3$, meaning the turbulence in the boundary layer strongly influenced the flow around the model; it suppresses the wake which leads to a lot shorter area of recirculation than low turbulence incoming flow would cause.
\end{abstract}

\section{Introduction}

In this contribution, research in the mechanics of fluids, that developed from the experimental testing of a model for civil engineering will be presented. Originally one of the often-investigated problems of civil engineering, wind loading on cooling towers, had been investigated. However, this paper is not going to deal with the cooling towers, it only originated there.

It was explained in [1] and [2], that the model of the cooling tower later started to be called a hyperboloidshaped model because an original civil-engineering problem solved in a classical way (wind loadings on cooling towers were measured and evaluated) later turned into a pure mechanics of fluids problem. After all the pressures on the model had been measured, Particle Image Velocimetry got involved and the velocity fields around the model were caught.

As e.g. Zdravkovich in [3] illustrates, the cooling towers problem can be remotely compared to a circular cylinder problem. To get a comparable picture of the flow, it is possible to come out of the mean flow topology of the cylinder, as depicted in [4] for instance. Therefore, in the flow around the hyperboloid object we could expect a horseshoe vortex and then, depending on the model geometry, a tip vortex, Kármán street vortex formation or arch vortex respectively and a very influential trailing vortex developed at the free end from the upwash in front of the object and on its sides, and the downwash at the back of the object. A detailed and thoroughly processed summary of these structures and under which conditions they appear, with many references to the relevant papers and literature was given in [5].

It might seem that this problem is already well described, however, there are many difficulties, when it comes to the model slightly differing from the simple geometry of regular and symmetric cylinder. The main difference is that we have a cylinder with variable diameter, thus the Reynolds number of the experiment varies from 40000 to 50000 . To further complicate the problem, we are talking about the model submerged in the boundary layer, which is to be explained later, so the velocity of incoming flow changes as the altitude of the model changes. This means that the boundary layer thickness developed on the model will vary as well. Additionally, the model remained covered by small grains from the civil engineering experiments, hence our last piece of known theory fails. Such rough surface forces the transition in the boundary layer on the model to occur at higher Reynolds numbers. On the other hand, the high rate of turbulence of incoming flow has opposite effect.

This phenomenon together with quite large high wall blockage and the low aspect ratio of the model, ensures the measurement with such model involved is filled with results different from the classic circular cylinder knowledge. Some background of the aspect ratios of the cylinders can be found in [6].

There is another separate object of discussion. According to [7], the shedding of vortices changes from a Kármán asymmetric type to a symmetric arch type at a geometrical aspect ratio of an object (cylinder) of 2.5. As the aspect ratio of our measured hyperboloid object varies

$\overline{\text { *Corresponding author: barraclough@vzlu.cz }}$ 
from 1.3 to 2.54 , the narrowest area of it could be measured with respect to this fact. Unfortunately, the equipment used for the experiment limited us, and this paper could not go deeper into this particular detail.

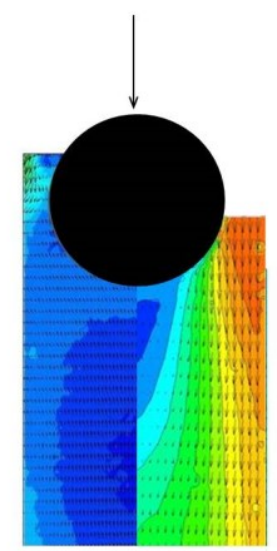

Fig. 1. A comparison of a wake behind a model in a Low Speed Wind Tunnel with a low rate of turbulence (left side of the wake) to the wake in a Boundary Layer Wind Tunnel with a high rate of turbulence (right side). The result is a field of time-averaged velocity field. The scalar map shows velocity $\mathrm{u} / \mathrm{U}$ from $-0,24$ to 1.

\section{Experimental Facility}

The experimental part was provided by means of 2D Particle Image Velocimetry (PIV). The measurement was provided at Reynolds numbers $\mathrm{Re}=40000 \div 50000$ with free stream velocity $U=5 \mathrm{~m} / \mathrm{s}$ measured independently on PIV by Prandtl probe. The tunnel used for the measurement was the Boundary Layer Wind Tunnel (BLWT) for simulating atmospheric boundary layer and built mainly for civil engineering measurements. It is equipped with a special surface helping the development of the boundary layer. This special surface is a foil with regularly placed bumps and, according to the standard for civil engineers [8], this equipment simulates a terrain of the $3^{\text {rd }}$ category: suburbs and unbroken forests. Detailed characteristics of this particular turbulent boundary layer, the tunnel itself, and the atmospheric boundary layer modelling can be found in report [9]. It has to be mentioned here, that the turbulence intensity falls from $30 \%$ close to the ground to $5 \%$ at $900 \mathrm{~mm}$ altitude. To complete the description of BLWT, the dimensions of the rectangular measurement area were $1.8 \times 1.5$ meters. For completeness, the second tunnel used for comparison was Low Speed Wind Tunnel of Eiffel Type with the low turbulence level (up to $1 \%$ ).

The model was printed using a $3 \mathrm{D}$ printer as a $0,32 \mathrm{~m}$ tall plastic object. The bottom part of the model consists of pillars and is penetrable for the flow. The model is hollow as well, so the suction effect of this bottom part will somehow influence the free end and the flow around it. The surface of the model is covered by grains of $0,6-0,7$ $\mathrm{mm}$ diameter. The roughness of the surface is one of the influencing parameters [10]. Effects of this parameter can be studied in [10], [11] and [12],. The ratio of the height of the model to the thickness of the modelled boundary layer in the BLWT is $1 / 3$. The velocity profile of the incoming flow in BLWT tunnel is depicted in Fig. 2.

The PIV experiment was provided in a classical way the measurement area was seeded with olive oil droplets produced by Laskin nozzles and a green laser light was used to illuminate the area. The $2 \mathrm{D}$ experimental part was provided with a laser with $\mathrm{Nd}$ :YAG crystals and low frequency $(15 \mathrm{~Hz})$ of the pulses. A double pulse mode was used, which means, that two consecutive pictures were captured within few tens of microseconds (usually 100) and the whole measurement lasted at least 60 seconds, so at least 900 of these double pictures were captured. For evaluation, a method called adaptive correlation was used with these parameters: final interrogation area was $64 \mathrm{x}$ 64 pixels, which represented $7-8.9$ x 7 - 8.9 square millimetres of real area. The capturing camera contained a chip with $2560 \times 2160$ pixels.

The main advantage of this method of getting experimental data is that the large area can be captured at one single moment. The main disadvantage is, apart from the amount of data and large storage media needed, that the strong illuminating light also strongly reflects from the surface. Meaning that the boundary layer around the model, with its thickness of few millimetres, can't be measured at the same moment as the large field of wake. The area close to the model is usually overexposed, and as the light reflects in all possible angles from the grains, the laser sheet there is useless.

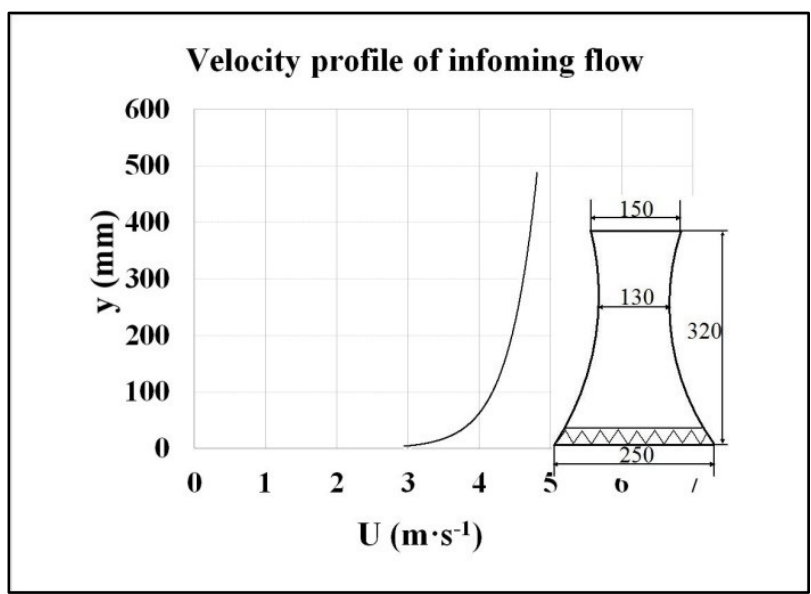

Fig. 2. The hyperboloid-shaped model with a vented base and its dimensions submerged in the boundary layer of depicted velocity profile.

\section{Measurements and results}

Some of the results were already presented in [1] and [2]. For this paper, special attention was paid to the measurement in the wake. The aim was to compare the influence of the turbulence intensity on a wake character. 


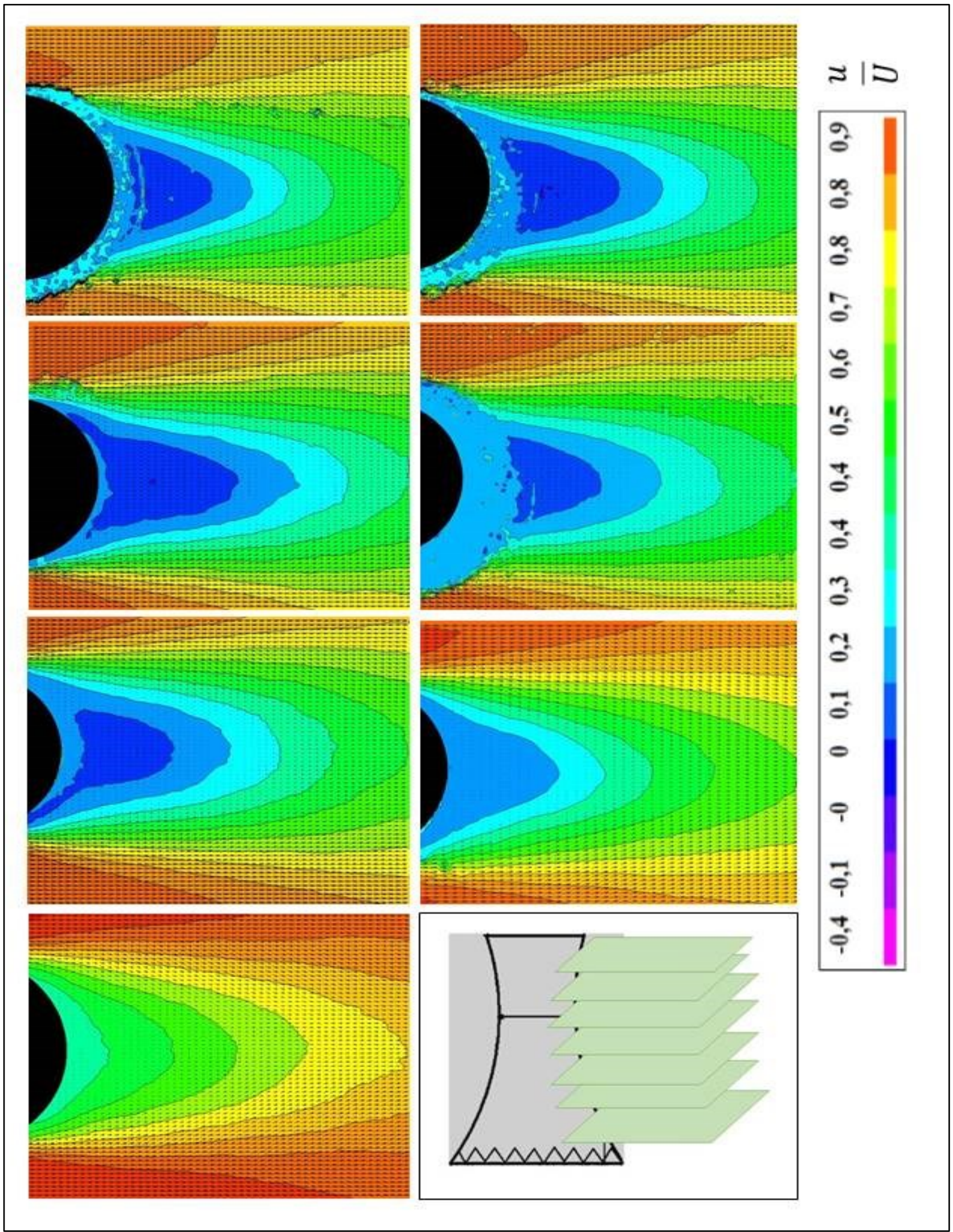

Fig. 3. The averaged velocity fields calculated in the planes parallel to the ground, varying altitudes; from top: $\mathrm{y} / \mathrm{L}=0,2$; 0,$34 ; 0,47 ; 0,59 ; 0,72 ; 0,84 ; 0,97$.

The areas $\mathrm{x} / \mathrm{D} \mathrm{x} z / \mathrm{D}$ of each field are as follows: $\mathrm{y} / \mathrm{L}=0,2: 2,73 \times 2,31 ; 0,34: 2,65 \times 2,23 ; 0,47: 2,53 \times 2,13 ; 0,59$ : $2,44 \times 2,05 ; \underline{0,72}: 2,34 \times 1,98 ; \underline{0,84}: 2,24 \times 1,89 ; \underline{0,97: 2,15 \times}$ 1,81 .

Dimension D represents minimum diameter of the model, L altitude of the model and velocity $U$ represents velocity of incoming flow. Bottom pictogram shows the measured areas. 


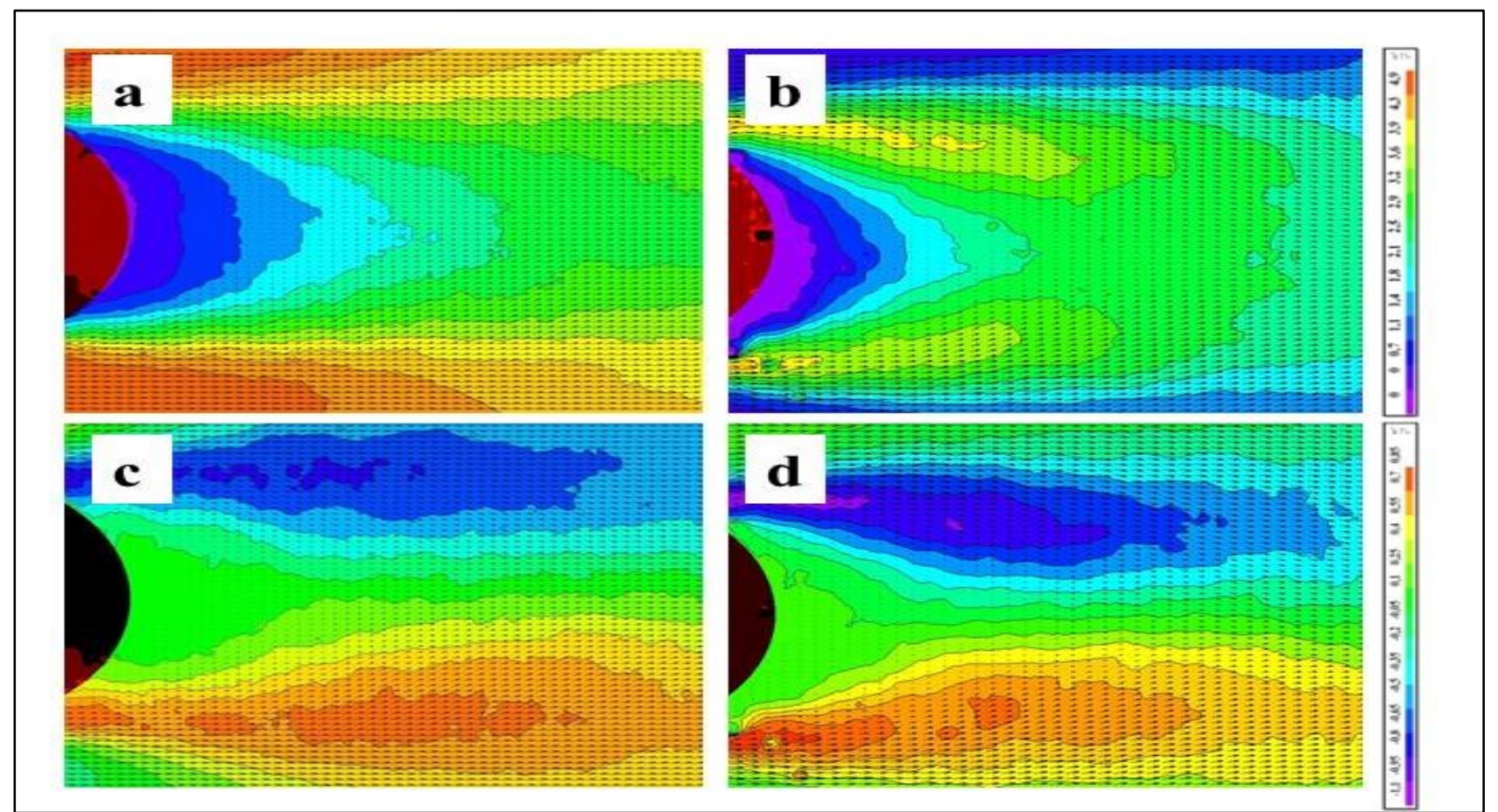

Fig. 4. Upper left and right: sum of variances (proportional to turbulent kinetic energy) at $\mathrm{y} / \mathrm{L}=0,97$ (a) and 0,84 (b) altitude and bottom left and right: covariances (proportional to Reynolds stresses) at 0,97 (a) and 0,84 (b) altitude. Dimension L represents the altitude of the model.
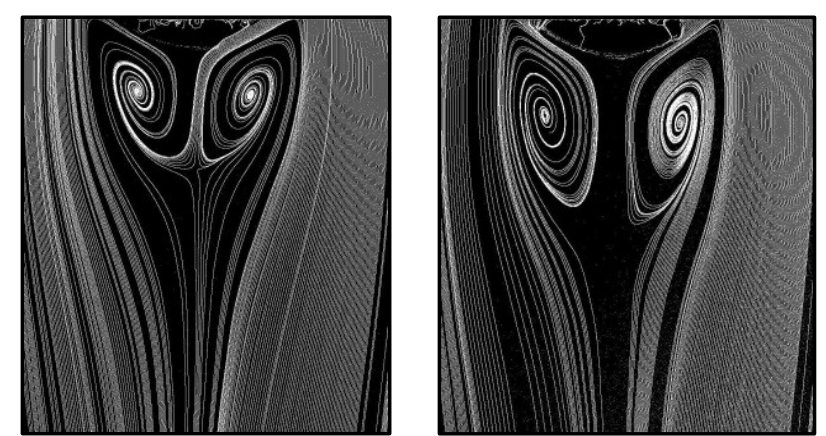

Fig. 5. The streamlines calculated from the averaged velocity field revealed two symmetric eddies (altitudes y/L 0,84 a 0,97 ).

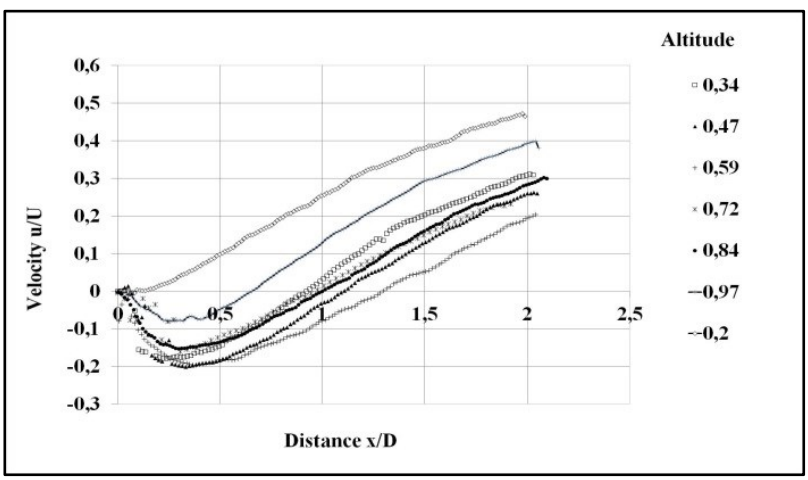

Fig. 6. $u / U$ velocity $x$-component profiles throughout wake at varying altitudes $y / L$. Dimension $D$ represents minimum diameter of the model, $\mathrm{L}$ altitude of the model and velocity $\mathrm{U}$ represents velocity of incoming flow.

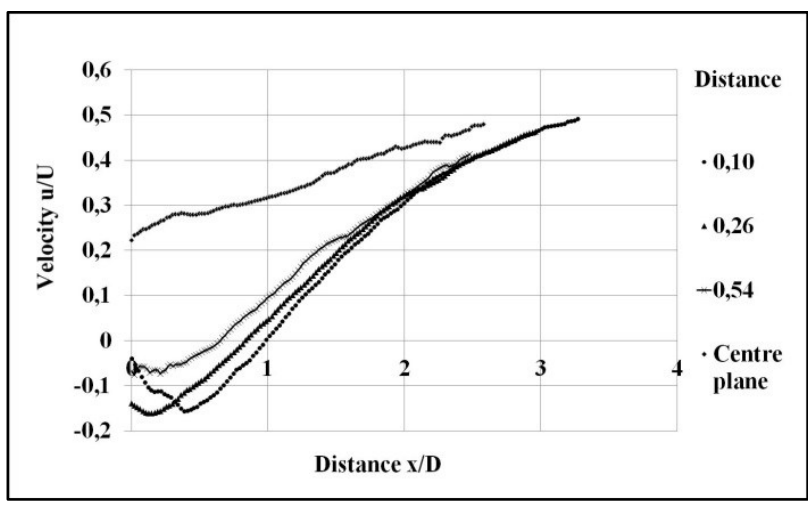

Fig. 7. $u / U$ velocity $x$-component profiles throughout the wake at various distances $\mathrm{z} / \mathrm{D}$ off the object axis plane as extracted at altitude $y / L=0,84$. Dimension $D$ represents minimum diameter of the model, velocity $U$ represents velocity of incoming flow.

The result of this phenomenon - influence of different turbulence intensity on the wake behind the model - can be seen in Fig. 1. The measuring plane for this particular test was set as parallel to the ground plane, at an altitude of $\mathrm{y} / \mathrm{L}=0,63$ from it. The influence of the turbulent intensity of the inlet flow on the wake is considerable, as it was already noticed in the abstract.

Afterwards, other measuring planes for more tests were parallel to the main one described above, in varying distances (see a pictogram in Fig. 3).

The statistically averaged velocity fields were calculated for each measurement, and then streamlines showing recirculating areas and possible location of most expected 3D structure, arch vortex, were evaluated. To confirm or disprove the existence of the arch vortex, other measurement planes for the wake were gained - parallel to the flow direction in the object axis and perpendicular to the ground this time (see a pictogram in Fig. 8). 

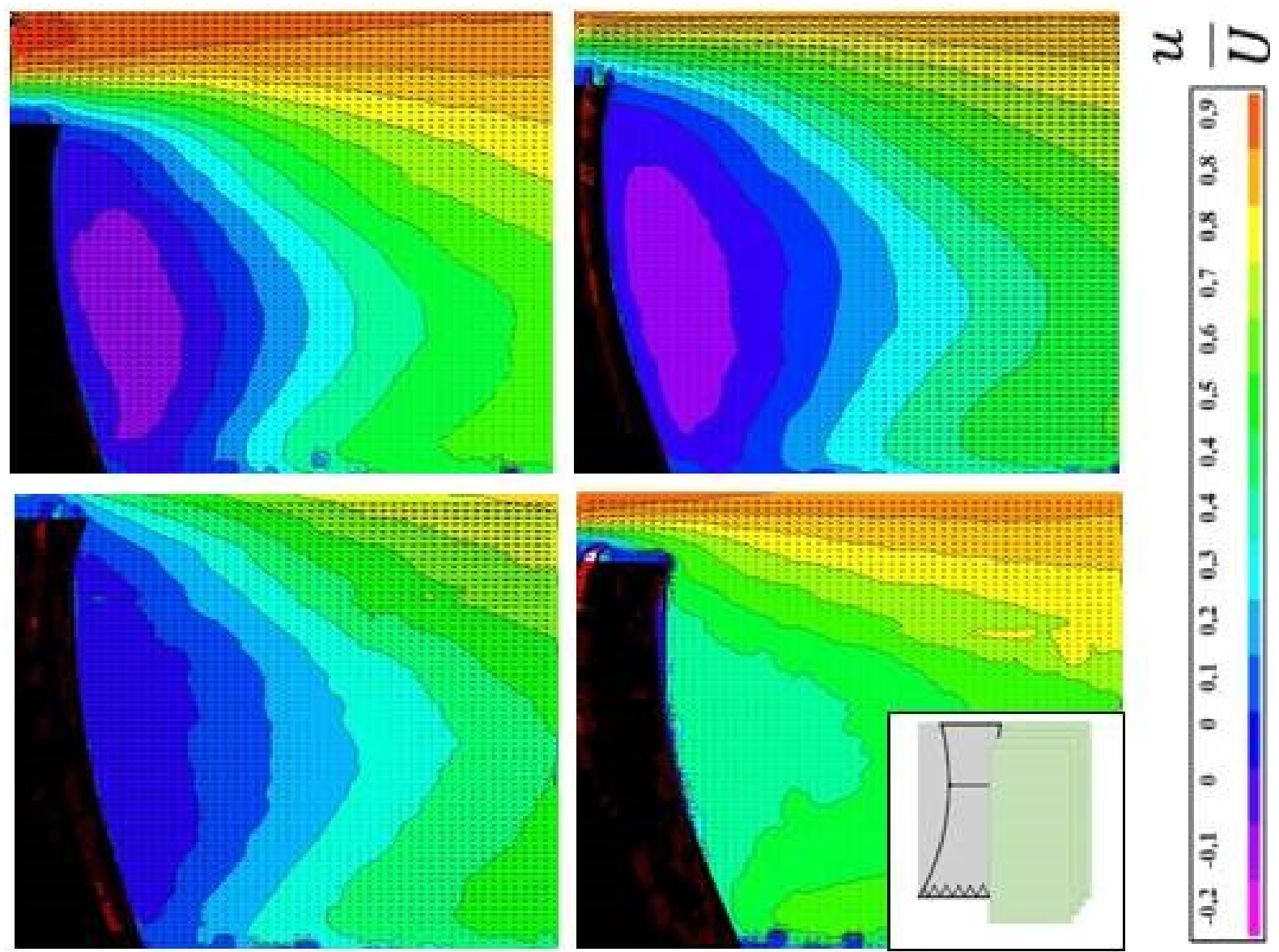

The scalar map shows x-component of velocity, a range $\mathrm{u} / \mathrm{U}$ is 0,2 to 0,9 . Bottom pictogram shows measured areas. The areas of fields of view $\mathrm{x} / \mathrm{D} \times \mathrm{y} / \mathrm{L}$ are as follows: $\mathrm{z} / \mathrm{D}=0: 1,3 \times 4,5$; $\mathrm{z} / \mathrm{D}=0,10: 1,2 \times 3,3 ; \mathrm{z} / \mathrm{D}=0,26: 0,93 \times 2,7 ; \mathrm{z} / \mathrm{D}=0,54: 0,9 \times 2,85$. the object axis plane, varying distances $\mathrm{z} / \mathrm{D}$ from a centre from top: $0 ; 0,10 ; 0,26 ; 0,54$. Dimension $L$ represents altitude of the model and velocity $U$ represents velocity of incoming flow.

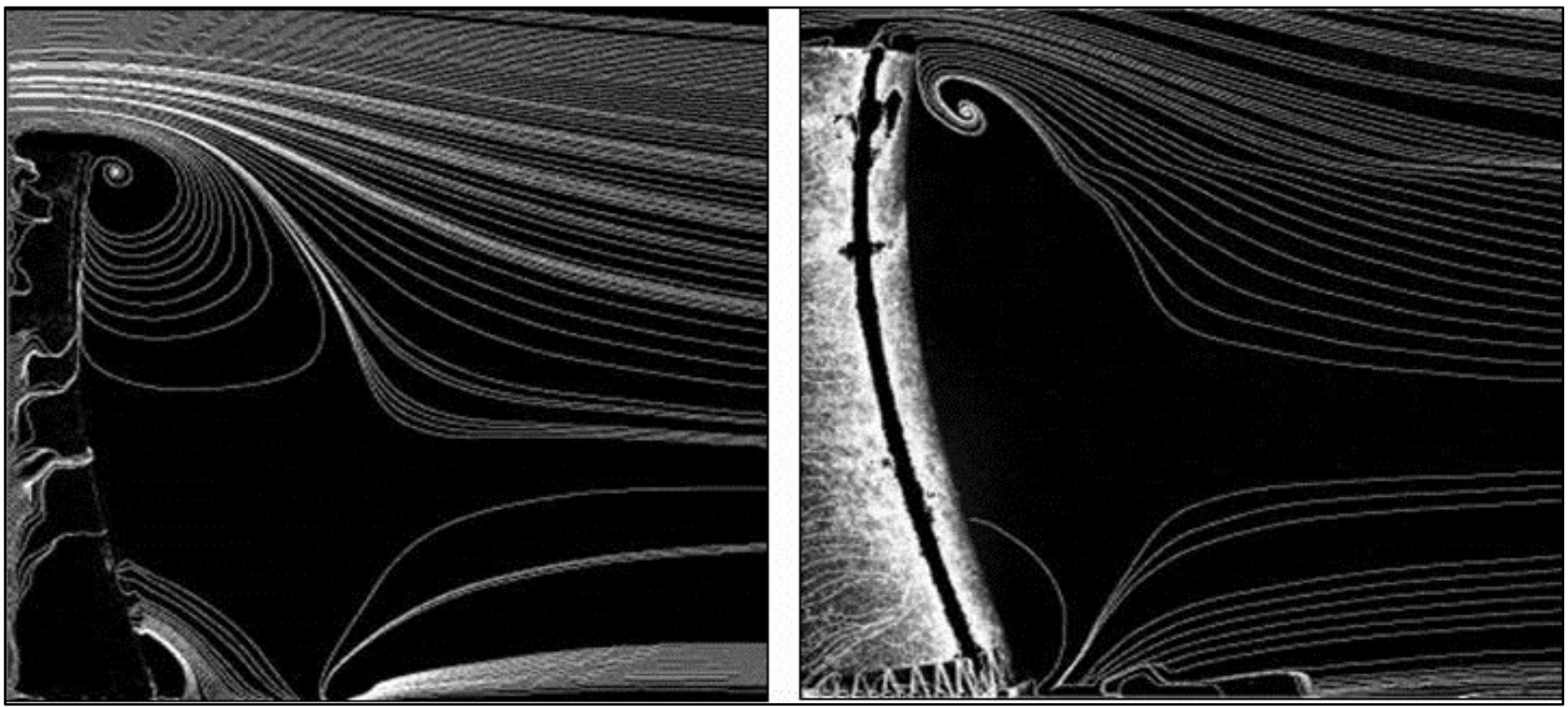

Fig. 9. Streamlines calculated from the averaged velocity field show the structure known as arch vortex (model-axis plane and $\mathrm{z} / \mathrm{D}=0,26$ off the axis plane). 


\section{Conclusion}

The wake of a hyperboloid-shaped model was measured in the wind tunnel with a modelled atmospheric boundary layer. The measurement was provided by means of $2 \mathrm{D}$ Particle Image Velocimetry. The ratio of the height of the model to the thickness of the modelled boundary layer in the BLWT was $1 / 3$, meaning that turbulence in the boundary layer strongly influences the flow around the model. Presented in Fig. 1, this parameter supresses the wake, leading to a much shorter area of recirculation than low turbulence incoming flow would cause.

The wake influenced by the high level of turbulence in the incoming flow was much shorter and followed turbulent separation; while the wake not disturbed by the high level of turbulence was longer and followed laminar separation. The turbulent separation is present in the subcritical range of the Reynolds number.

From averaged velocity fields (Fig. 3), one can see the size of the recirculating area in different planes. In addition, if the sum of variances and covariance pictures, which are proportional to turbulent kinetic energy and Reynolds stresses respectively, are displayed (Fig. 4), one can surmise a shear layer border and an area of boundary layer separation.

The single illustrations in Fig. 5 indicate the run of the vortex filament in a perpendicular plane to the ground. It actually copies the shape of the recirculating area. The idea obtained from the measurement is that the arch vortex is also bent in the $\mathrm{x}$ direction. This could be the contribution of the three dimensions of the model, or the boundary layer, or both equally.

An interesting, but not surprising, result was given by extracting the $\mathrm{x}$-component velocity plots from the central part of the wake (Fig. 6 and 7). The zero on the x-axis was taken in the surface of the model. A closer view to the single graphs detects, that the running of the velocity at various altitude levels differs and there is no recirculating area close to the top. In the near wake, up to the $\mathrm{x} / \mathrm{D}=0,3$ behind the cylinder, the results seem to be chaotic. There is also no regularity. We cannot just say the closer to the ground the slower the flow is.

Looking at the results in Fig. 9, the streamlines calculated from the averaged velocity field revealed supposed 3D structure - the desired arch-shaped vortex. Its upper part appears at the dimensionless altitude between $\mathrm{y} / \mathrm{L} 0,84$ and 0,97 . Other planes measured parallel to this one were in the distances of $\mathrm{z} / \mathrm{D}=0,1$ and 0,26 , and still showed possible vortex filament. It is obvious that at a distance of $\mathrm{z} / \mathrm{D}=0,26$ the arch already veers towards the ground.

\section{Acknowledgements}

The support from the Project No. CZ.2.16/3.1.00/21569 Centre $3 \mathrm{D}$ Volumetric Anemometry is gratefully acknowledged.

This work was supported by the Grant Agency of the Czech Technical University in Prague, grant No. SGS17/074/OHK2/1T/12.

The research was performed with financial institutional support from the government budget through the Ministry of Industry and Trade of the Czech Republic.

\section{References}

1. V. Barraclough, J. Novotný, P. Šafařík: Study of Flow around Model of Cooling Tower by Means of 2D Particle Image Velocimetry Measurement. Experimental Fluid Mechanics, EPJ Web of Conferences, 01/2017 (2016).

2. V. Barraclough, J. Novotný, P. Šafařík: Experimental Investigation of 3D Vortex Structures in Wake of Hyperboloid-Shaped Model, Topical Problems of Fluid Mechanics, Conference Proceedings (2017).

3. M.M. Zdravkovich: Flow around Circular Cylinders. Vol.2: Applications, Oxford University Press, (2003).

4. R.J. Pattenden, S.R. Turnock, X. Zhang.: Measurements of the Flow over a Low-Aspect-Ratio Cylinder Mounted on a Ground Plane, Experiments in Fluids 39: 10-21. (2005).

5. J.L. Heseltine: Flow around a Circular Cylinder with a Free End. A Thesis for the Degree of Master of Science, The Department of Mechanical Engineering, University of Saskatchewan, Canada (2003).

6. D. Sumner, J.L. Heseltine: Tip Vortex Structure for a Circular Cylinder with a Free End, Journal of Wind Engineering and Industrial Aerodynamics 96, (2008).

7. H. Sakamoto, M. Arie: Vortex Shedding from a Rectangular Prism and a Circular Cylinder Placed Vertically in a Turbulent Boundary Layer. Journal of Fluid Mechanics, pp. 147-165, vol. 126, (1983).

8. European Standard EN 1991-1-4:2005 Eurocode 1: Actions on Structures.

9. M. Jirsák: Physical Principles of Work with Boundary Layer Wind Tunnel. An Internal Report (in Czech), Aeronautical Research and Test Establishment, Plc., Prague, Czech Republic (2012).

10. M.M. Zdravkovich: Flow around Circular Cylinders, Oxford University Press (1997).

11. M.M. Zdravkovich: Conceptual Overview of Laminar and Turbulent Flows Past Smooth and Rough Circular Cylinders. Journal of Wind Engineering and Industrial Aerodynamics, 33, 53-62 (1990).

12. R. Merrick, G.T. Bitsuamlak: Control of Flow around a Circular Cylinder by the Use of Surface Roughness: A Computational and Experimental Approach, www.researchgate.net (2014). 